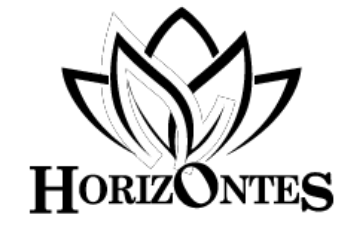

www.revistahorizontes.org

\title{
La composición escrita. Aspectos considerados por el docente para su evaluación
}

The written composition. Aspects considered by the teacher for their evaluation

A composição escrita. Aspectos considerados pelo professor para avaliação

ARTÍCULO ORIGINAL

\section{Carmen Zenaida Hernández \\ zenaida2464@gmail.com}

Unidad Educativa “Creación Guacarapa”, Miranda, Venezuela

Unidad Educativa “Colegio La Milagrosa”, Miranda, Venezuela

\section{RESUMEN}

En la presente investigación se planteó como objetivo general describir los aspectos de la escritura que considera el docente cuando evalúa la composición escrita de los estudiantes. El enfoque para esta investigación se ajustó al paradigma cualitativo. Los sujetos de la investigación fueron docentes de 4to, 5to y 6to. Grado de la Unidad Educativa Colegio La Milagrosa, ubicada en Guarenas - estado Miranda. Las técnicas empleadas fueron la entrevista semiestructurada y la observación directa o participativa y como instrumento el guion de entrevista. Entre las conclusiones se resalta que los docentes, sujetos de esta investigación, no evalúan el tipo de texto ni su estructura, ni la composición escrita como proceso, sino como producto final, no supervisan a los participantes para que estos revisen sus escritos y para que, a su vez, ellos verifiquen si plasmaron sus ideas en forma correcta.

Palabras clave: Composición escrita, evaluación de textos escritos, proceso de revisión

\begin{abstract}
In the present investigation, the general objective was to describe the aspects of writing that the teacher considers when evaluating the written composition of the students. The approach for this research was adjusted to the qualitative paradigm. The research subjects were 4th, 5th and 6th teachers. Degree of the Educational Unit College La Milagrosa, located in Guarenas Miranda state. The techniques used were the semi-structured interview and direct or participatory observation and as an instrument the interview script. Among the conclusions it is highlighted that the teachers, subjects of this research, do not evaluate the type of text or its structure, nor the written composition as a process, but as a final product, they do not supervise the participants so that they review their writings and so that, in turn, they verify if they have expressed their ideas correctly
\end{abstract}

Key words: Written composition, evaluation of written texts, revision process 


\section{RESUMO}

Na presente investigação, o objetivo geral foi descrever os aspectos da escrita que o professor considera ao avaliar a composição escrita dos alunos. A abordagem desta pesquisa foi ajustada ao paradigma qualitativo. Os sujeitos da pesquisa foram os $4^{\circ}, 5^{\circ}$ e $6^{\circ}$ professores. Graduado pela Unidade Educacional Colégio La Milagrosa, localizada em Guarenas - Miranda. As técnicas utilizadas foram a entrevista semiestruturada e observação direta ou participativa e como instrumento o roteiro da entrevista. Dentre as conclusões, destaca-se que os professores, sujeitos desta pesquisa, não avaliam o tipo de texto ou sua estrutura, nem a composição escrita como processo, mas como produto final, não supervisionam os participantes para revisar seus escritos e para que por sua vez, eles verificam se expressaram suas ideais corretamente.

Palavras-chave: Composição escrita, avaliação de textos escritos, processo de revisão

\section{INTRODUCCIÓN}

El desempeño de los estudiantes, sus actividades y comportamiento escolar, sus composiciones escritas y concreciones verificables en sus cuadernos o en cualquier otro material escolar tangible reportan información válida para constatar el rendimiento, el aprendizaje y las debilidades que presentan en su desempeño escolar, así como rasgos de su vida socioemocional.

Entre las actividades escolares que se realizan en la escuela y que permiten dar cuenta del nivel de aprendizaje y de las dificultades que demuestran los estudiantes en su vida escolar destaca la escritura. Esta actividad reviste importancia capital en el currículo de los diferentes niveles del sistema educativo y, más aún, en educación primaria, por cuanto las composiciones escritas constituyen un espacio en el cual los estudiantes expresan la comprensión que tienen del mundo que se presenta ante ellos en el ámbito escolar.

La escritura es uno de los medios de expresión que utiliza el ser humano para enunciar sus ideas, pensamientos, sentimientos y necesidades. Al respecto, Arteaga Quintero, Cova Jaime y Álvarez (2010) plantean que:

La escritura es una herramienta empleada en todos los ámbitos de la vida, es un acto de comunicación que se utiliza con distintos propósitos, que no necesariamente responden a exigencias académicas, pero que requiere de la activación de distintos procesos mentales ( $p$. 27).

Por su parte, Cassany (2002) sostiene que algunos de los valores o cimientos más significativos de la civilización contemporánea clavan sus raíces en la escritura y en su potencial comunicativo.

A través de las composiciones escritas se logra dejar plasmada toda una gama de información que brinda oportunidades de descubrir nuevos caminos para alcanzar grandes conocimientos. Cruz (2002) destaca que "la escritura se considera un arte y el proceso compositivo; una lucha para dar sentido a la existencia, para aprender, para expresarse, para elevar nuestras vidas por cuanto son dignas de escritura" (p. 30).

Podría afirmarse que la escritura es un medio comunicativo que permite dar rienda suelta al pensamiento y exaltar el deseo de enunciar lo que se desea expresar en un momento dado. En este orden de ideas, Serrano y Madrid (2002) expresan que "el escritor cuando compone se obliga a realizar un conjunto de operaciones tales como reflexionar sobre sus ideas, reconsiderar, analizar y reorganizar lo que desea comunicar" (p. 88). 
Es indudable que uno de los medios del que dispone el ser humano para comunicarse es a través de las composiciones escritas. En tal sentido, como bien lo indica Ríos (2002) "constantemente necesitamos comunicarnos por escrito con otras personas para hacerles saber nuestros pensamientos y sentimientos; así, en la vida cotidiana, necesitamos escribir cartas, postales, notas y diarios; en el proceso educativo debemos elaborar composiciones, monografías, informes, ensayos y tesis" (p. 75).

Desde esta perspectiva, se considera que la función de la lengua escrita ofrece grandes oportunidades para que todo individuo se comunique, lo que indica que la misma está inmersa en la vida. Igualmente, según Franco y Noriega (2002):

Desde el punto de vista cognitivo, la escritura es un proceso intelectual en el que se requiere una representación jerárquica de ideas, de una aceptación de las reglas del sistema de la lengua y sobre todo de un manejo de convenciones socioculturales ( $p$. 110).

Es importante señalar que la adquisición del arte de la escritura se podría considerar indispensable para la asimilación de los conocimientos y el desarrollo de algunas aptitudes en el ámbito escolar. De la misma forma, González (2009) considera:

Es prudente preguntarse cuáles son los conceptos que deben considerarse para caracterizar, presentar y orientar la lengua escrita en el aula porque en la medida en que se conoce más del tema en cuestión, las posibilidades para especificar el tratamiento de la lectura y la escritura con los aprendices son mayores (p. 7).

De acuerdo con lo planteado, se explica que desde el punto de vista lingüístico, el proceso de escritura requiere del uso de una serie de pautas que guíen al escritor de manera que sea capaz de reproducir sus ideas en forma escrita.

González (2009) indica "la escritura es un acto lingüístico, reflexivo. Exige el uso de palabras en un contexto lingüístico y en un contexto social para expresar ideas con un propósito definido" (p 13). Es a partir de ahí donde comienza el camino de la revisión de cada proceso que permite interpretar $y$ evaluar los aportes de las composiciones escritas de los estudiantes, ya que las mismas constituyen evidencias significativas de las actividades académicas dentro del aula.

Para Camps (2006) en la escuela se crean espacios de escritura en las que los alumnos deben atender a las características de los destinatarios para ajustar los escritos a las necesidades que la comunicación demanda.

En el ámbito escolar, es el docente el encargado de orientar y guiar a los estudiantes en los diferentes niveles, para que construyan textos escritos con coherencia, ajustados a las normas gramaticales, con informaciones relevantes $\mathrm{y}$, especialmente, con sentido tanto para quien los escribe $y$ para quien los leerá.

Las composiciones escritas, como un instrumento de gran relevancia en la vida de todo estudiante, testimonia la historia del ambiente áulico y recopila informaciones de índole curricular, didáctica, evaluativa además de reflejar la relación interactiva entre padres y educadores.

En la redacción de una composición escrita confluyen contenidos, proceso de escritura, audiencia, propósito; selección de 
vocabulario y de ideas; organización de las ideas, ortografía y puntuación; gramática y sintaxis (Lunar, 2007).

Toda composición escrita requiere de un proceso de revisión que valore los resultados, oriente al estudiante hacia la correcta escritura y corrija a tiempo los errores cometidos, tales como: omisión de vocales, consonantes, sílabas, palabras, fallas ortográficas, entre otros; donde se oriente al alumno sobre aquellos desaciertos que comete cuando realiza alguna actividad escrita.

Las composiciones escritas constituyen evidencias significativas de las actividades académicas dentro del aula. En estas, se puede observar parte del pensamiento infantil, hábitos, nivel de responsabilidad y seguimiento de instrucciones. Sin embargo, no se sabe si los docentes otorgan al proceso de revisión y evaluación la importancia que amerita. Pareciera que el docente no emplea la evaluación de estos instrumentos para analizar el proceso de aprendizaje de sus educandos, para detectar fortalezas y ciertas debilidades o deficiencias.

De allí que el propósito de esta investigación fuera describir los aspectos de la escritura que considera el docente cuando evalúa la composición escrita de los estudiantes del subsistema de Educación Primaria, específicamente en los grados 4to, 5to y 6to (Hernández, 2014). Esto debido a que la experiencia permite inferir que el docente, al momento de planificar actividades vinculadas con las composiciones escritas que deben realizar los estudiantes, considera muy poco los aspectos relacionados con este proceso; tales como la planificación, la textualización y, especialmente, el proceso de revisión, lo cual podría redundar en las deficiencias de escritura que son cada vez más alarmantes.

\section{La evaluación}

Desde tiempos muy remotos, la evaluación está inmersa en la vida cotidiana de todo ser humano, ya que cada una de las actividades que realiza las somete a un continuo proceso de evaluación, lo que permite conocer los resultados de todo cuanto hace, con el fin de determinar el grado de satisfacción del cumplimiento de los objetivos propuestos.

Al respecto, Córdoba (2006) expresa que "la palabra evaluación, como muchas otras, posee múltiples significados, significados que van a depender de las diferentes perspectivas y contextos desde los cuales se aborde el término" (p. 3).

Esto implica que todo proyecto que emprende un individuo lo hace con la finalidad de alcanzar una meta, pero para que eso sea posible, se requiere seguir paso a paso una serie de lineamientos que le permita evaluar cada aspecto importante de los objetivos alcanzados. Así como también se debe evaluar el procedimiento y la búsqueda de las diferentes alternativas que conduzcan a un aprendizaje significativo, dependiendo del contexto y del punto de vista de cada participante.

En este orden de ideas, Mateo (2000) afirma que "se trata de una praxis transformadora que para incidir en profundidad, precisa activar los resortes culturales, sociales y políticos más relevantes que en los contextos en que actúa" (p. 21).

Cabe destacar que desde que el hombre existe, hace uso de la evaluación para conocer y definir las capacidades que tiene para lograr un objetivo propuesto, para saber las fallas que presenta y buscar las posibles soluciones a los conflictos que se le presenten en el transcurso de la realización de alguna actividad emprendida, ya que evaluar implica conocer las debilidades y potencialidades que cada uno posee. 
Por lo anterior, Stufflebeam (2002) postula que la evaluación es el proceso de identificar, obtener y proporcionar información útil y descriptiva acerca del valor y mérito de las metas, la planificación, la realización y el impacto de un objeto determinado.

En tal sentido, se consideran tres conceptos elementales en el proceso de evaluación, tales como: la fiabilidad, la viabilidad y la validez, los cuales se relacionan entre sí, permiten precisar y obtener los resultados que se buscan en una investigación. En el caso del proceso que se debe seguir en las composiciones escritas, es necesaria una continua evaluación que permita valorar los diferentes momentos, como lo son la planificación, la transcripción y la revisión y así detectar las habilidades y dificultades que se presentan en dicho proceso.

Por tanto, la evaluación debe enrumbarse hacia el perfeccionamiento de cada una de las actividades que se llevan a cabo en la vida cotidiana de todo ser, sobre todo en este caso, las actividades que son realizadas en el ambiente áulico, tomando en cuenta que todo estudiante debe seguir un régimen de evaluación que brinde las pautas requeridas para conformar la formación integral que cada uno recibe.

Por su parte, Gutiérrez (2003) destaca que la evaluación del aprendizaje es uno de los factores que más influye en el interés de los estudiantes por aprender y por el propio proceso de aprendizaje. En consideración a lo señalado, el docente debe apropiarse de los intereses y necesidades de sus estudiantes para aplicar una evaluación de calidad que ofrezca una gama de posibilidades y un encuentro para compartir saberes, donde los estudiantes logren alcanzar las competencias de los contenidos desarrollados, tomando en cuenta que el proceso de evaluación debe ser participativo donde están involucrados todos los agentes inmersos en las actividades.

Gutiérrez (2003) afirma que la información que aporta la evaluación permite que el docente realice una observación continua y pueda tomar decisiones en cuanto a los apoyos que requiere el estudiante.

Un ambiente áulico sin una continua evaluación sería un espacio sin una respuesta a las situaciones que se presentan en el día a día escolar; es carecer de las informaciones que se requieren para detectar a tiempo las fallas o aciertos que presentan los estudiantes, incluso, para conocer si los docentes están aplicando las estrategias necesarias que contribuyan al enriquecimiento de conocimientos de los participantes. Se evalúa para continuar con una estrategia de trabajo o para rediseñar un proyecto emprendido, se evalúa para darle sentido a los resultados del quehacer humano y estar consciente del rumbo que debe y quiere seguir.

En la evaluación debe considerarse el qué, el para qué y el cuándo se evalúa de modo que puede ser evaluación diagnóstica o inicial; evaluación formativa o de proceso y evaluación sumativa o final; integradora o de resultado. La evaluación en cada uno de sus momentos es necesaria, ya que brinda la oportunidad al evaluador de saber de dónde debe partir para emprender una práctica pedagógica que atienda las necesidades de la población que se va a evaluar (Ruiz, 2002).

Por otra parte, de entre los modelos de evaluación existentes, para efectos de esta investigación, se adoptó el modelo de evaluación CIPP de Daniel Stufflebeam, pues en esta propuesta la evaluación se concibe como un proceso dinámico de toma de decisiones de muy diversos tipos. En este caso, se pone énfasis en la necesidad de que la enseñanza de la escritura se desarrolle en las aulas de forma que el profesor pueda 
intervenir durante el proceso como mediador y proporcionar el andamiaje que los estudiantes necesitan para resolver los múltiples problemas que se presentan durante la composición escrita y para poder observar y emitir juicios sobre las producciones escritas, con el objetivo de mejorar el proceso de enseñanza y de aprendizaje en este campo (Camps y Ribas, 2006).

\section{La composición escrita}

La composición escrita es una actividad comunicativa que permite al escritor expresar sus ideas, sentimientos, inquietudes y necesidades. Como lo señala Ríos (2002) "la composición escrita es un proceso cognitivo complejo mediante el cual la persona traduce sus representaciones mentales: ideas, pensamientos, sentimientos e impresiones en discurso escrito coherente, en función de hacérselos llegar a una audiencia de una manera comprensible" (p. 166).

Al respecto, Fraca (2007) expresa que "el arte de la escritura es un compromiso con la palabra y esta se hace sentir en el otro, en el lector, por eso debemos ser muy cuidadosos en el buen decir y en el buen escribir" (p.10). Bien es cierto que la composición escrita permite dejar ver el pensamiento del que escribe, ya que la escritura es un arte que expresa el pensamiento humano.

Serrano y Madrid (2002) sostienen que: "La escritura se considera un arte y el proceso compositivo; una lucha para dar sentido a la existencia, para aprender, para expresarse, para elevar nuestras vidas por cuanto son dignas de escritura. " (p. 30).

Como se puede ver, la composición escrita representa para la sociedad una potencialidad de gran importancia para el desarrollo del pensamiento expresivo, donde se requiere de la comprensión necesaria para su práctica y desarrollo.
Se considera que actualmente en los sistemas educativos, toda persona debe lograr como competencia básica la composición escrita (Albarrán y García, 2010). En la composición escrita el pensamiento se desplaza a través de las letras que forman cada palabra, cada párrafo, cada mensaje que lleva a un lector una información de algún tema determinado.

En el día a día de toda persona está inmersa la escritura como medio de comunicación útil y necesaria en la vida social de todos los ciudadanos. Como dice Caldera (2006) "la escritura es una tarea compleja porque es un proceso que involucra distintas demandas cognitivas, lingüísticas, metacognitivas, motrices y socioculturales en una actividad que implica dedicación, paciencia y práctica" (p. 92).

Para adquirir todo tipo de conocimientos se requiere de una serie de pasos los cuales guían al aprendiz a lograr un aprendizaje significativo y de un proceso de adaptación y asimilación a cada uno de los descubrimientos que va logrando, como lo es el caso del ejercicio de la escritura.

Desde muy temprana edad se debe involucrar el proceso de escritura en el sistema de vida de cada quien, porque escribir es una forma de hablar y comunicar lo que en un momento dado desea expresar. El proceso de escritura es actividad que se practica a través de la imitación; es decir se aprende más que todo en las escuelas (Sánchez, 2009).

Lo que indica que es necesario que en el ambiente áulico se dedique especial atención a la enseñanza de la escritura, con la finalidad de facilitar el proceso de composición escrita, donde se ofrezcan las herramientas necesarias para lograr habilidades y destrezas en el desarrollo de las composiciones escritas. En algunos espacios académicos se ha dejado a un lado las prácticas en el proceso de composición 
escrita (Horrillo, Álvarez, Requena y Romo, 2006).

Partiendo de esta última idea, se considera que es de vital importancia que en los centros educativos se brinden todas aquellas posibles oportunidades para el buen desarrollo de la composición escrita, donde se valore los aspectos que permiten dar sentido y significado al proceso de la escritura, tomando en cuenta que la escritura es una creación literaria, una acción donde el escritor deja plasmado sus ideas de lo que piensa, de lo que siente, de lo que desea expresar en sus composiciones escritas.

Por tanto, la composición escrita es de gran importancia para lograr los objetivos propuestos en el ámbito educativo y para el crecimiento del nivel cognitivo de todo estudiante, ya que si estos leen y escriben adquieren un alto nivel cognitivo que favorece su desarrollo y crecimiento como ser social capaz de enfrentarse al día a día con el mejor andamiaje para atravesar los puentes del conocimiento.

Es aquí donde los educadores juegan un papel protagónico en la vida de cada participante, porque son los docentes las personas más idóneas para ofrecer las herramientas necesarias que permiten adquirir las habilidades y destrezas requeridas para el proceso de la composición escrita.

La escritura es la voz del pensamiento escrito, es la palabra que no se pronuncia fonéticamente, son las expresiones que se dejan moldeadas para comunicarse con el lector.

\section{METODOLOGÍA}

El enfoque seleccionado para esta investigación fue el cualitativo. La investigación cualitativa exige el reconocimiento de múltiples realidades y trata de capturar la perspectiva del investigador. Este enfoque permitió abordar el problema desde varias dimensiones, considerando especialmente que la escritura es una práctica social que involucra complejos procesos de elaboración y representación de ideas. En cuanto al método cualitativo empleado en este estudio fue el método hermenéutico. Adema,

Esta investigación estuvo enmarcada dentro de un diseño de investigación de campo de carácter descriptivo e interpretativo, según los objetivos propuestos en este estudio porque los datos fueron recogidos directamente de la realidad; específicamente entre los docentes $\mathrm{y}$ estudiantes del subsistema de educación primaria de la Unidad Educativa Colegio La Milagrosa, ubicada en Guarenas, Estado Miranda. De allí que los participantes de esta investigación fueran tres (3) docentes de dicha institución, los cuales fueron sometidos a un proceso de observación que permitió analizar cómo realizan el proceso de revisión de las composiciones escritas de sus estudiantes. Para ello, se seleccionaron las composiciones escritas de los niños que cursan cuarto, quinto y sexto grado en el mencionado colegio. Estas composiciones escritas o corpus permitieron considerar aspectos importantes y necesarios para realizar un mejor análisis, de acuerdo con los fines que persiguió la investigación.

Se consideraron los tres últimos grados de Educación Primaria porque es allí donde el estudiante inicia el aprendizaje en la composición de textos escritos; pues en los primeros grados debe aprender letras, sílabas, palabras, frases, oraciones e iniciarse en la ortografía para que, en los grados superiores desarrolle la destreza de componer textos escritos.

Las técnicas e instrumentos, al igual que los procedimientos y estrategias que se utilizaron; se corresponden con el enfoque 
metodológico seleccionado. En este caso, las técnicas se concentraron básicamente en la observación directa y la entrevista semiestructurada. El instrumento empleado fue el guión de entrevista.

El procedimiento empleado consistió en aplicar la entrevista a los tres docentes seleccionados, con el fin de describir los aspectos de la escritura que estos consideraban cuando evaluaban la composición escrita de los estudiantes de 4to, 5to y 6to grado del subsistema de Educación Primaria en la Unidad Educativa Colegio La Milagrosa. Cada entrevista tuvo una duración de 30 minutos aproximadamente.

En la Tabla 1, se incluye la definición de cada una de las categorías y subcategorías de análisis.

Tabla 1. Categorías apriorísticas y su de definición con las subcategorías

\begin{tabular}{cl}
$\begin{array}{c}\text { Categorías } \\
\text { apriorísticas }\end{array}$ & \multicolumn{1}{c}{ Definición conceptual } \\
ASPECTOS DE LA & $\begin{array}{l}\text { Se refiere a las herramientas } \\
\text { lingüísticas que caracterizan a la } \\
\text { ESCRITURA }\end{array}$ \\
$\begin{array}{l}\text { lengua escrita y que se debes } \\
\text { seguir al redactar un texto escrito. }\end{array}$
\end{tabular}

\section{MACROESTRUCTURA}

Estructura de contenido: generación y organización de ideas y vocabulario

\section{SUPERESTRUCTURA}

Estructura esquemática: tipología textual y órdenes del discurso

\section{MICROESTRUCTURA}

Estructura gramatical: coherencia cohesión, signos de puntuación, ortografía y sintaxis

\section{PROCESO DE COMPOSICIÓN ESCRITA}

4.1. Planificación

4.2. Transcripción

4.3. Revisión

\section{RESULTADOS}

Para dar respuesta al objetivo de la presente investigación, en la Tabla 2 se muestran, por cada categoría, las subcategorías y los hallazgos o evidencias seleccionadas de las entrevistas a los docentes. Al inicio de cada hallazgo extraído se coloca y destaca un código que indica a cuál sujeto pertenece la información seleccionada, se codificó de la siguiente manera: D4 (docente de 4to. grado), D5 (docente de 5to. grado) y D6 (docente de 6to. grado). 
Tabla 2. Hallazgos en la categoría aspectos formales de la escritura

\section{SUBCATEGORÍAS}

MACROESTRUCTURA

Estructura PROPIEDADES generación y organización de ideas y vocabulario

SUPERESTRUCTURA

MICROESTRUCTURA

PROCESO DE COMPOSICIÓN ESCRITA
Estructura esquemática: tipología textual y órdenes del discurso

Estructura gramatical: coherencia, cohesión, signos de puntuación, ortografía y sintaxis

1. Planificación

2. Transcripción

3. Revisión

\section{HALLAZGOS}

D4. Les cuesta mucho generar ideas pero yo los dejo solos es para que se acostumbren a tener sus propias ideas. En cuanto al vocabulario ellos pretenden escribir como hablan.

D5. A veces intercambian ideas entre ellos o me preguntan y yo los ayudo.

D6. Organizan las ideas pero al principio les cuesta mucho, aunque a veces escriben muy mal y con un vocabulario que da pena.

D4. Los alumnos escriben textos narrativos, textos instruccionales, textos explicativos, entre otros. Depende de la actividad que se le solicite.

D5. Producen textos informativos, narrativos y explicativos.

D6. Escriben textos expositivos, textos informativos y textos narrativos.

D4. Yo corrijo el uso de los verbos, la presencia del sujeto y el sentido que le dan a los escritos asignados en cada actividad.

D5. Les corrige la coherencia, la cohesión, las reglas ortográficas y la adecuación del texto escrito en cada alumno.

D6. Corrijo todos aquellos aspectos gramaticales que forman la estructura de un texto escrito y le dan sentido al mismo.

D4. Luego de realizar cada trabajo escrito, yo les indico dónde están sus errores para que ellos corrijan y mejoren sus trabajos escritos.

D5. Una vez que el estudiante me hace entrega de sus trabajos escritos, se les corrige de manera que los mismos modifiquen su escritura.

D6. Todo alumno debe hacer las correcciones que el docente le indique para que se logre el objetivo. 


\section{Subcategoría macroestructura}

Se refiere al contenido semántico global que constituye el sentido de un texto. La macroestructura está representada por el núcleo informativo fundamental, que es el tema del que trata el texto. Sus propiedades están determinadas por las ideas y su organización en el escrito.

Según los hallazgos explicitados en el cuadro 3, los estudiantes de 4to., 5to. y 6to. Grado no reciben orientación por parte de los docentes a fin de que puedan generar ideas en torno al tema específico que abordarán en sus composiciones escritas. Las respuestas dadas por los docentes en las entrevistas son muestras de que estos no acompañan a los alumnos para guiarlos en sus escritos ni registran los resultados de los mismos. En escasas ocasiones, ejercitan la lluvia de ideas y su organización jerárquica para, posteriormente plasmarlas en un escrito. Más concretamente, los docentes en el nivel de Educación Primaria orientan muy poco a sus estudiantes en la generación y organización de ideas antes de producir un texto escrito.

$\mathrm{Si}$ en un texto, la secuencia de oraciones carece de macroestructura, el escrito es percibido como una continuidad de enunciados incoherentes $\mathrm{y}$, por lo tanto, no llega a constituirse como texto. La macroestructura, en este sentido, es un mecanismo de coherencia textual (Van Dijk, 1980). Lo anterior fundamenta la importancia de que los docentes ejerciten la coherencia global cuando sus estudiantes se enfrenten a la tarea de escribir.

Los docentes, sujetos de esta investigación, no evalúan la organización de las ideas ni el vocabulario.

\section{Subcategoría superestructura}

Representa la forma como se organiza la información en el texto, esto es, la estructura textual formal. La superestructura define el esquema adoptado para abordar el tema textual.

Las propiedades de la superestructura de un texto están dadas por la tipología textual y órdenes del discurso. Es aquí donde el docente aborda los tipos de textos y su estructura. Sin embargo, en los grados investigados, se observa que los docentes suponen que todos los alumnos manejan los tipos de textos y su estructura. En la entrevista solo informaban que sus estudiantes producen textos narrativos, informativos, instruccionales, explicativos y expositivos. Los mismos docentes no distinguen la diferencia entre tipos de textos y órdenes del discurso; por tanto, cómo orientan a los discentes.

Los docentes, sujetos de esta investigación, no evalúan el tipo de texto ni su estructura.

\section{Subcategoría microestructura}

Es una sinopsis de las ideas o conceptos planteados en el texto según su orden de importancia. Es aquí donde se deben identificar ideas principales y secundarias, conceptualizar, realizar una síntesis a través de un análisis del título y analizar los ejemplos relevantes para comprender el tema.

Las propiedades de la microestructura responden a la estructura gramatical del texto; esto se logra con la coherencia, la cohesión, el correcto uso de los signos de puntuación, la ortografía y la sintaxis.

Los sujetos de la investigación plantearon que, en las composiciones escritas de sus alumnos, evalúan: los verbos, la coherencia, la cohesión, la ortografía y la adecuación del texto.

\section{Subcategoría proceso de composición escrita}

Se define como un proceso cognitivo complejo mediante el cual la persona traduce sus representaciones mentales: ideas, 
pensamientos, sentimientos e impresiones en discurso escrito coherente, para hacerlos llegar a una audiencia de manera comprensible (Ríos, 2000).

Las propiedades del proceso de composición están determinadas por tres subprocesos: planificación, transcripción y revisión. La enseñanza de la escritura se debe desarrollar en las aulas de manera que el docente intervenga en el proceso como guía que oriente a los estudiantes en cómo abordar el proceso. En las entrevistas, quedó en evidencia que el docente no evalúa la composición escrita como proceso, sino como producto final ya acabado.

Todo este análisis e interpretación sirvieron para describir los aspectos de la escritura que considera el docente cuando evalúa la composición escrita. Las docentes únicamente se limitan a corregir algunos errores ortográficos, sin aclarar al estudiante lo que debe hacer; no participan en el proceso de la composición de sus alumnos. La escritura como proceso no es evaluada, pues no toman en cuenta los pasos que siguen los estudiantes en sus composiciones escritas y, finalmente, se puede afirmar que no se trabaja con borradores previos como parte del proceso de construcción del texto escrito. Los únicos aspectos de la composición escrita que consideran cuando evalúan a sus estudiantes son los signos de puntuación y la ortografía; es decir, no toman en cuenta ni la macroestructura, ni la superestructura del texto escrito y mucho menos las etapas del proceso de la composición escrita.

Por otra parte, en las figuras 1,2 y 3 , se muestra el resultado de la triangulación que se hizo comparando la respuesta de las tres docentes en cuanto a las categorías apriorísticas que fueron consideradas. Esta triangulación se hizo a partir de lo señalado por Martínez (1999), quien dice que esta consiste en determinar ciertas intersecciones o coincidencias a partir de diferentes apreciaciones y fuentes informativas o varios puntos de vista del mismo fenómeno." (p. 98).

Finalmente, en la Tabla 3 se presenta el resultado de la triangulación entre los datos recogidos en los instrumentos, la teoría reportada en el marco referencial y la interpretación realizada. 

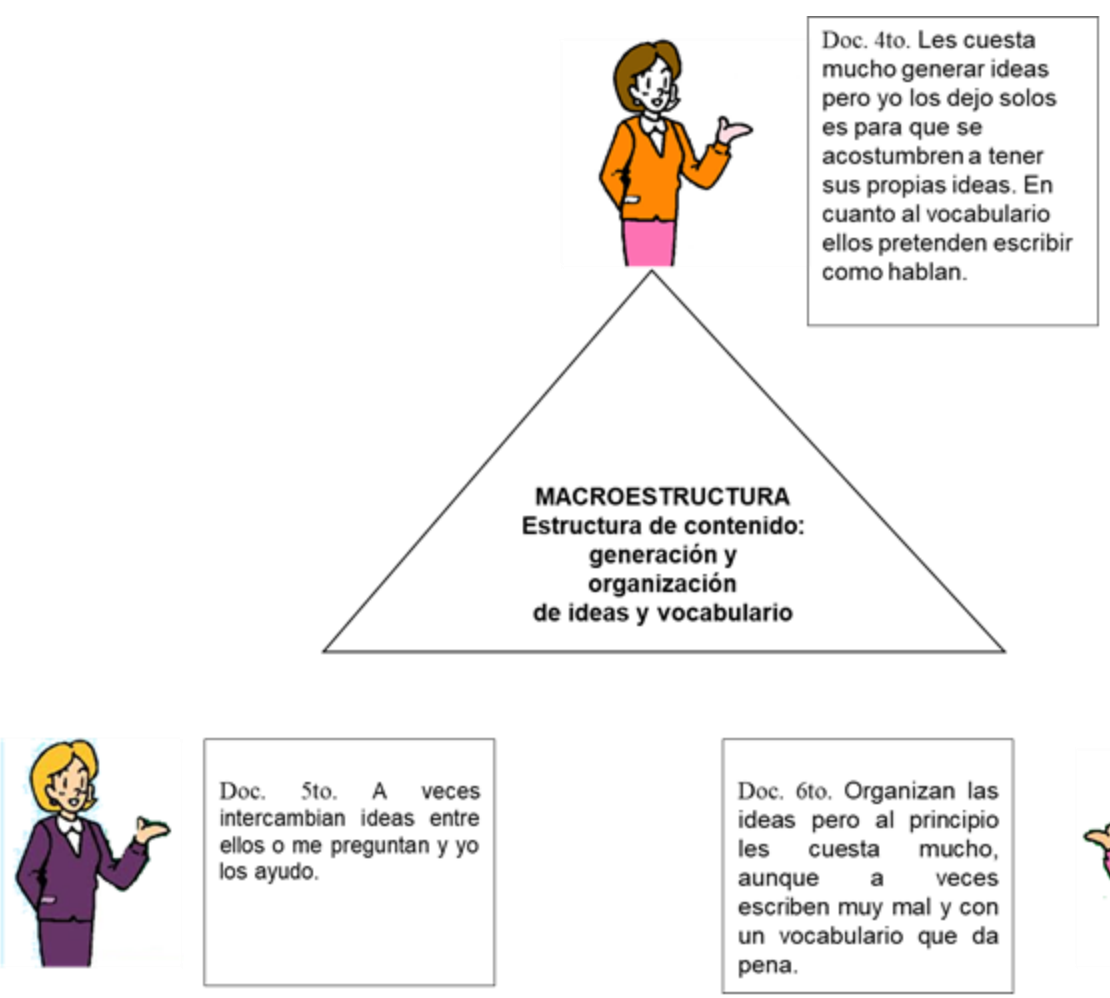

Doc. 6to. Organizan las ideas pero al principio les cuesta mucho, aunque a veces escriben muy mal y con un vocabulario que da pena.

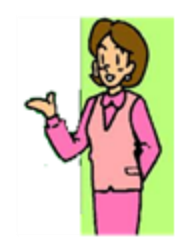

Figura 1. Macroestructura
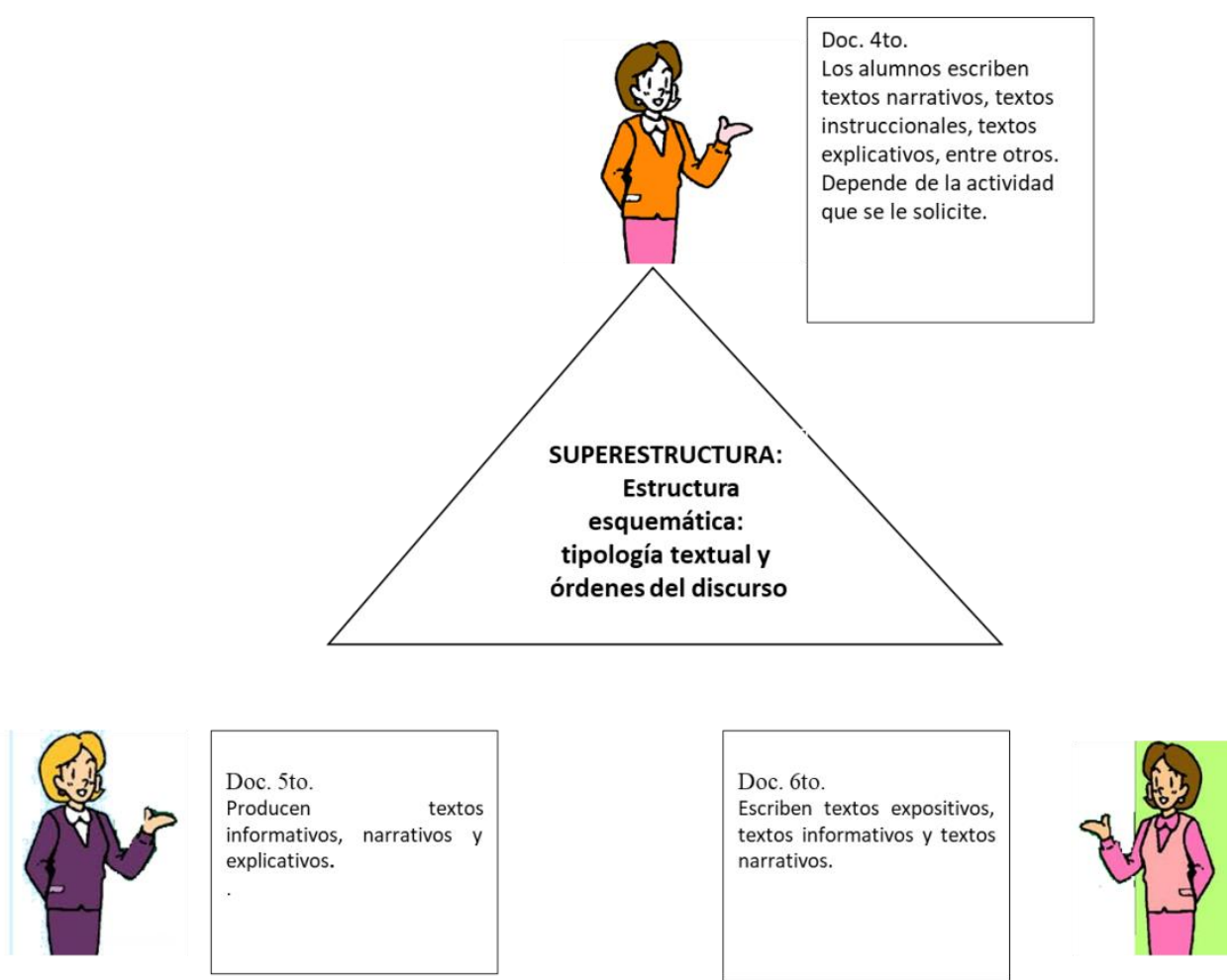

Figura 2. Superestructura

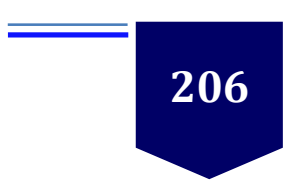



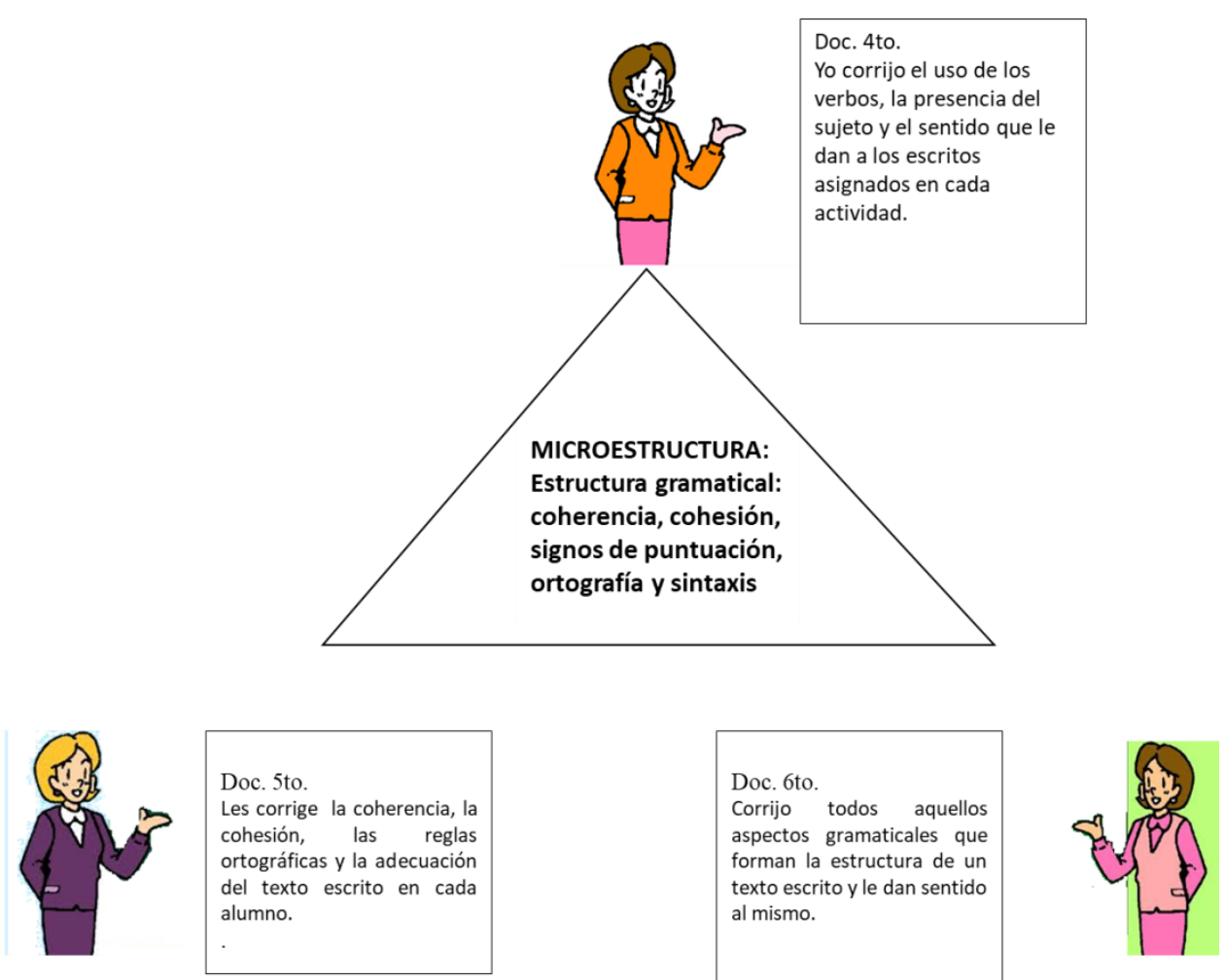

Figura 3. Microestructura
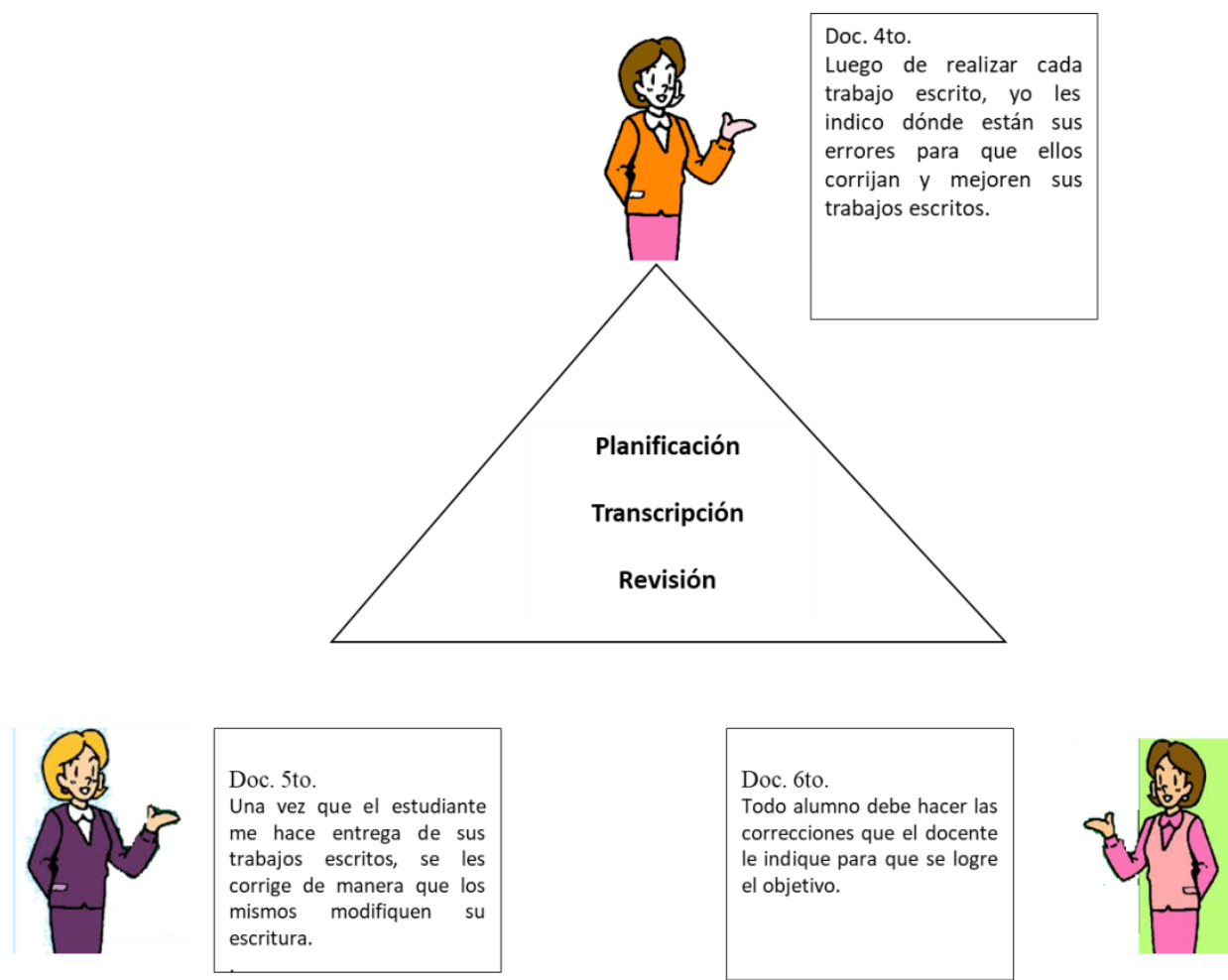

Figura 4. Planificación, transcripción y revisión 
Tabla 3. Triangulación de la Categoría: Aspectos formales de la escritura

\section{EVIDENCIAS}

Los docentes de los tres grados investigados no evalúan la organización de las ideas, el vocabulario empleado, los lineamientos establecidos según los órdenes del discurso (texto narrativo, descriptivo, expositivo o argumentativo), la coherencia, cohesión, sintaxis ni la composición como proceso en sus tres etapas.

Solamente corrigen, en algunos casos, los signos de puntuación y la ortografía; además de la legibilidad de algunas palabras.

\section{SUSTENTO TEÓRICO}

Sánchez (2009) explica que "se requiere del uso de la habilidad de redactar, de lectura, comprensión y la expresión oral para componer un escrito. Escribir es quizá la habilidad lingüística más compleja durante el proceso de composición" (p. 356).

Fernández, Núñez y Romero (2009) argumentan que es principalmente la escuela la institución responsable de que los escolares adquieran los niveles más elevados en el dominio de la lengua escrita.

El que escribe debe atender a aspectos de ortografía, de uso del léxico, de arreglo sintáctico, de comunicación de significados, de estilo y organización textual, y sobre ellos, orquestar y coordinar una producción que tiene mucho de creativa $\mathrm{y}$ original (Hayes $\mathrm{y}$ Flower, 1981).

\section{INTERPRETACIÓN}

Todo docente debe tener en cuenta que escribir es una habilidad compleja y para ello, quien escribe tiene que dominar los aspectos formales de la escritura, que incluye la macroestructura, la superestructura y la microestructura de un texto.

Sin embargo, se evidencia que los docentes, sujetos de esta investigación, solo se circunscriben a revisar dos de los aspectos de la estructura gramatical: la ortografía y los signos de puntuación, los cuales pertenecen a la microestructura del texto. Los demás aspectos, relacionados a la macroestructura y a la superestructura, son obviados y por consiguiente el alumno no avanza en la tarea de aprender y apropiarse de correcto y complejo manejo de la lengua escrita.

\section{CONCLUSIONES}

Para describir los aspectos de la escritura que consideran los docentes cuando evalúan las composiciones escritas de sus estudiantes, se analizaron las correcciones que realizaron a los textos escritos:

Los resultados evidenciaron que los docentes no evalúan la organización de las ideas, el vocabulario, tampoco acompañan a los alumnos para guiarlos en sus escritos ni registran los resultados de estos. En cuanto a la superestructura, estos docentes no consideran el tipo de texto ni su estructura y, en relación con la microestructura, los sujetos de la investigación solo corrigen los signos de puntuación y la ortografía. Po tanto, resulta necesario señalar que las docentes únicamente se limitan a corregir algunos errores ortográficos, sin aclarar al estudiante lo que debe hacer; no participan en el proceso de la composición de sus alumnos.

La escritura como proceso no es evaluada, pues no toman en cuenta los pasos que siguen los estudiantes en sus composiciones escritas $y$, finalmente, se puede afirmar que no se trabaja con borradores previos como parte del proceso de construcción del texto escrito; es decir, no toman en cuenta ni la macroestructura, superestructura del texto escrito, ni las etapas del proceso de la composición escrita.

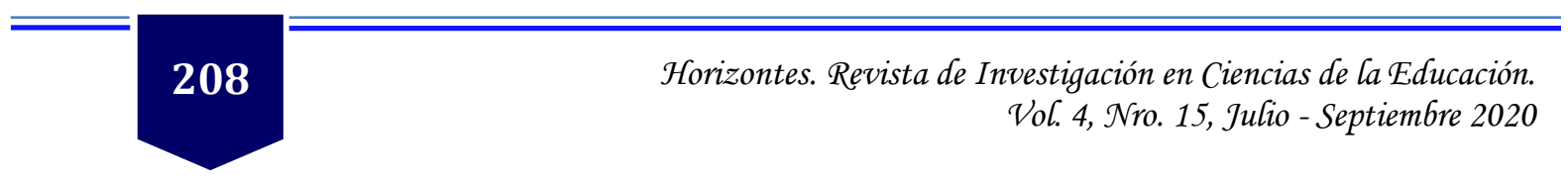


REFERENCIAS

Albarrán, M. y García, M. (2010). El proceso de enseñanza de la composición escrita adaptado a la evolución del aprendizaje de la escritura de los estudiantes. Didáctica de la Lengua y Literatura ISSN: 1130-0531. Recuperado de http://www.ucm.es/BUCM/revistas/edu/11 300531/articulos/DIDA1010110015A.PDF

Arteaga Quintero, M., Cova Jaime, Y. y Álvarez, N. (2010). Comprensión y producción de textos escritos. Teoría y práctica. Caracas: UPEL - IPMJMSM

Caldera, R. (2006). Proposiciones teóricas para la enseñanza-aprendizaje de la escritura en educación básica. Recuperado de http://www.saber.ula.ve/bitstream/1234 56789/17637/2/articulo4.pdf

Camps, A. (2006). Escribir para aprender: una visión desde la teoría de la actividad. Trabajo de investigación en la Universidad Autónoma de Barcelona. Recuperado de http:/cedw.org/es/admin/uploads/congr esos/12/act/7/campsana

Camps, A., y Ribas, T. (2006). Evolución de un texto escrito durante una secuencia de enseñanza y aprendizaje de la redacción: incidencia de las pautas de revisión. En: Pérez Pereira, M. (Ed.): Estudios sobre la adquisición del castellano, catalán, eusquera y gallego. Santiago de Compostela, Universidad de Santiago de Compostela, pp. 655-669

Cassany, D. (2002). De lo analógico a lo digital; el futuro de la enseñanza de la composición. Revista Textura, 1(1), 15 - 19

Córdoba, F. (2006). Revista Iberoamericana de Educación, número 39/7. Recuperado de http://www.rieoei.org/1388.htm

Cruz, J. (2002). Modalidades de desarrollo de la escritura en entornos escolares. Revista Textura, 1(I), 29 - 30

Fraca, L. (2007). La cultura escrita y la pedagogía integradora en el desarrollo de la discursividad infantil. En: A. Bolívar (Comp.) Análisis del discurso ipor qué y para qué? Caracas: Editorial CEC, S. A. Los libros de El Nacional
Franco y Noriega. (2002). La autocorrección como estrategia para la formación de escritores autónomos. Revista Textura, 1 (I), 110

González, N. (2009). Escribir para seguir escribiendo. Revista Onza Tigre y León, octubre $\mathrm{N}^{\circ} 30$

Gutiérrez, O. (2003). Enfoques y Modelos Educativos Centrados en el Aprendizaje. Estado del Arte y propuestas para la operativización en las instituciones de educación superior nacionales. Madrid: Morata

Horrillo, A., Álvarez, G., Requena, R. y Romo, M. (2006). Propuesta de mejora en la composición escrita. Recuperado de http://www.juntadeandalucia.es/averroe s/ cepco3/competencias/lengua/primari a/LA_COMPOSICION_ESCRITA.pdf

Lunar, L. (2007). El portafolio: estrategia para evaluar la producción escrita en inglés por parte de los estudiantes universitarios. Escuela de educación Facultad de Humanidades y Educación. U.C.V. Recuperado de http://wwwscielo.org.ve/ scielo.php?pid

Martínez, M. (1989). "El método hermenéutico-dialéctico en las ciencias de la conducta". Anthropos, 18, 85-111

Martínez, M. (1999). La Nueva Ciencia. Su desafío, lógica y método. México: Trillas

Mateo, J. (2000). La evaluación educativa, su práctica y otras metáforas. Barcelona: ICE Universidad de Barcelona

Ríos C., P. (2000). La aventura de aprender. Caracas: Cognitus

Ríos, P. (2002). Elementos para una didáctica de la producción escrita. Revista Textura, 1(I), $78-79$

Ruiz, L. (2002). Evaluación. Tipos de Evaluación. Investigación y Postgrado, 2 (2), p. 16 - 23

Sánchez, D. (2009). La expresión escrita en la clase de ELE. Estrategias de enseñanza y aprendizaje del español. Madrid: Morata 
Serrano, S. y Madrid A. (2002). Aprender a revisar para producir textos. Creación y análisis de situaciones didácticas. Revista Textura, 1(I), 85 - 89
Stufflebeam, D. L. (2002). Evaluación sistemática. Guía teórica y práctica. Barcelona, España: Paidós 